\title{
Erratum to: Trade-Offs in Automatic Provenance Capture
}

\author{
Manolis Stamatogiannakis ${ }^{1(\bowtie)}$, Hasanat Kazmi ${ }^{2}$, Hashim Sharif ${ }^{2}$, \\ Remco Vermeulen ${ }^{1}$, Ashish Gehani ${ }^{2}$, Herbert Bos ${ }^{1}$, and Paul Groth ${ }^{3}$ \\ ${ }^{1}$ Computer Science Institute, Vrije Universiteit Amsterdam, Amsterdam, \\ The Netherlands \\ \{manolis.stamatogiannakis, r.vermeulen, h. j.bos\}@vu.nl \\ 2 SRI International, Menlo Park, USA \\ \{hasanat.kazmi, hashim. sharif, ashish.gehani\}@sri.com \\ 3 Elsevier Labs, Amsterdam, The Netherlands \\ p.groth@elsevier.com
}

\section{Erratum to: \\ Chapter 3: M. Mattoso and B. Glavic (Eds.) Provenance and Annotation of Data and Processes DOI: 10.1007/978-3-319-40593-3_3}

The text that was missing is:

Acknowledgments. This material is based upon work supported by the National Science Foundation under Grant IIS-1116414. Any opinions, findings, and conclusions or recommendations expressed in this material are those of the authors and do not necessarily reflect the views of the National Science Foundation. 